
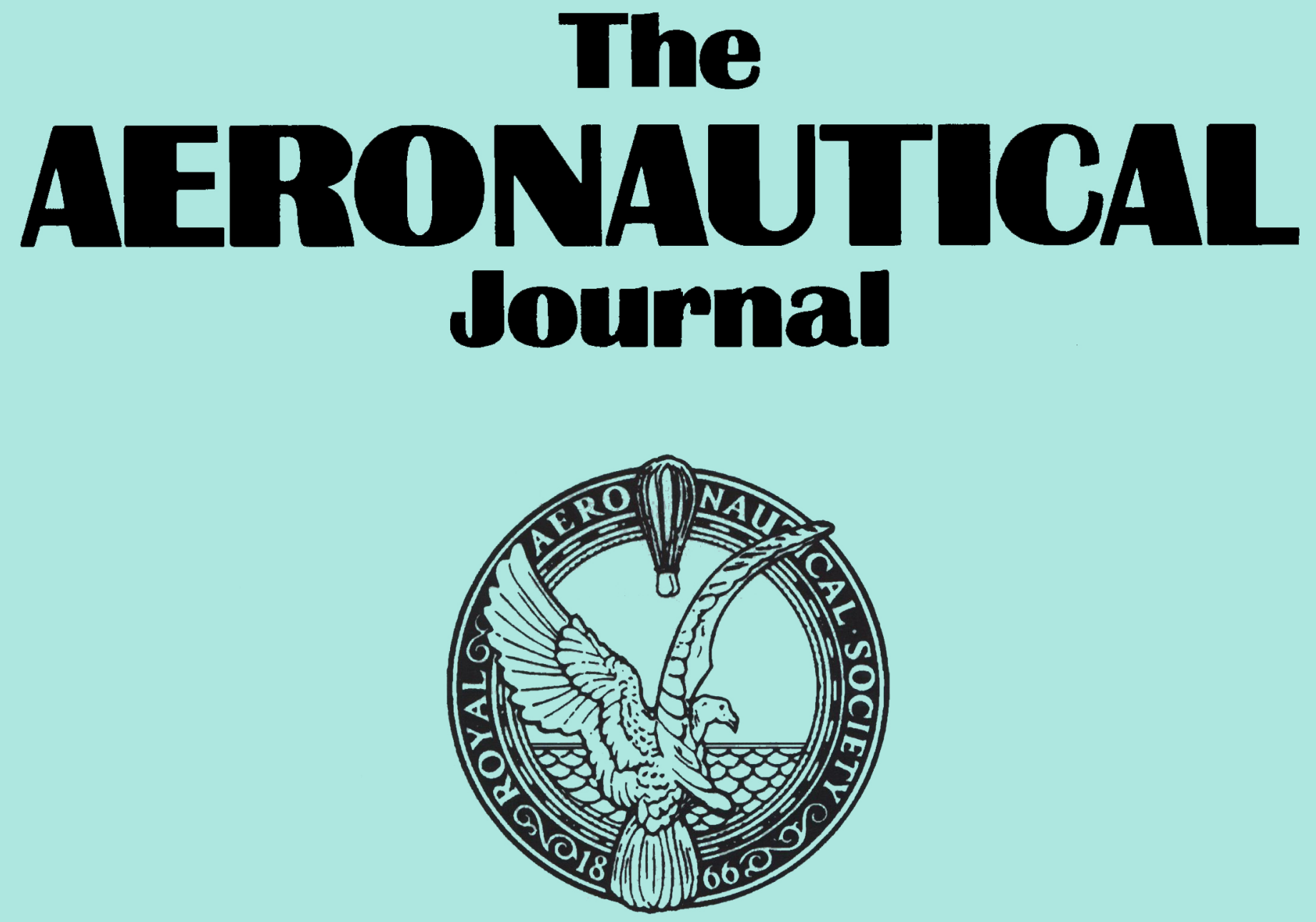

\title{
Contents
}

\section{P.H. Summerfield}

The world of regional aircraft - challenges and opportunities

G. luso

Turbulence structure manipulation in a channel flow by outer layer devices

\section{A.G. Barnes}

Modelling requirements in flight simulation 


\section{THE AERONAUTICAL JOURNAL}

\section{Aims and scope}

The aims and scope of The Aeronautical Journal are intended to reflect the objectives of the Society as expressed in the Charter of Incorporation. Briefly these are to encourage and foster the advancement of all aspects of aeronautical and space science. Thus the topics of the Journal include all those which are covered by the various Sections and Groups of the Society such as fluid mechanics and aerodynamics, propulsion, structures and materials, rotorcraft, astronautics and guided flight, dynamics and control, aeromarine technology, aviation medicine, air transport, test flying, flight simulation, air law, management studies, history of aviation and humanpowered flight. Thus papers are solicited on all aspects of research, design and development, construction and operation of aircraft and space vehicles. Papers are also welcomed which review, comprehensively, the results of recent research developments in any of the above topics. 


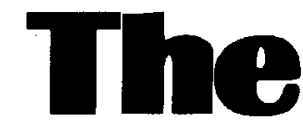

Volume 98 Number 980 December 1994
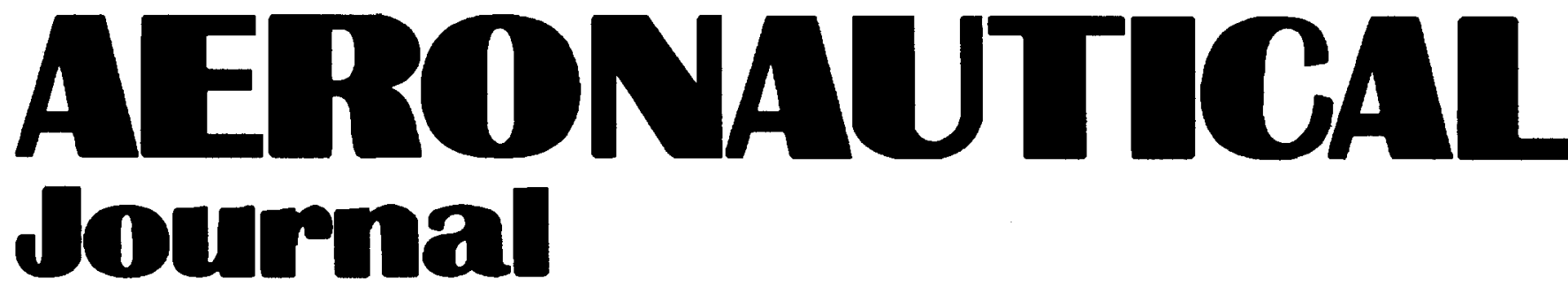

\title{
Editorial Advisory Board
}

\author{
Chairman: Dr E.W.E. Rogers
}

Editor

B.F. Baldwin, BSc, Associate Member RAeS

Deputy Editor

S.M. Penney, BEng, Grad RAeS

Editorial Assistants

C.S.C. Male, BSc(Eng), Grad RAeS

I.R. Sheppard, BEng, Grad RAeS

RAeS Director

R.J. Kennett, FIMgt, FInstD, FCIT, FRSA, AFAIAA, FRAES

Published by

The Royal Aeronautical Society

4 Hamitton Place, London WIV $0 B Q$.

Tel: 071-499 3515 .

Fax: 071-499 6230.

Advertisements

Chris Marot/Trevor Hornshaw

Marot \& Co

3 Albion Buildings

1 Back Hill, London EC1R 5EN

Tel: $071-2783686$

Fax: 071-837 2764

Reproduction of any of the papers published in this journal is not

permitted without the written consent

of the Editor.

The content does not necessarily express the opinion of the Council.

Printed by

Manor Park Press,

Unit 7, Highfield Industrial Estate,

Edison Road, Hampden Park,

Eastbourne.

Subscriptions: $£ 195$ a year, post free.

RAeS members: £22 a year.

Single copies, including back numbers: £24.

Non-member subscriptions from

The Royal Aeronautical Society

Publications Subscriptions Department,

PO Box 139, Tonbridge,

Kent TN9 1EW, UK.

Tel: 0732770823.

Fax: 0732361708

ISSN: 0001-9240

The Royal Aeronautical Society is a registered charity.

\section{Aerodynamics}

Professor P.W. Bearman, Department of Aeronautics, Imperial College

\section{Aerospace medicine}

Air Vice Marshal P. Howard, formerly RAF Institute of Aviation Medicine

Air traffic control and simulation

G.C. Howell, former Civil Aviation Authority chief scientist

Air transport

Professor R. Doganis, Department of Air Transport, Cranfield Institute of Technology

Airworthiness

R. Ashford, European Joint Aviation Authority

Avionics and systems

Professor D. McLean, Department of Aeronautics \& Astronautics, University of Southampton

P.A. Hearne, GEC-Marconi Avionics

Computational fluid mechanics and propulsion

Professor P. Stow, Rolls-Royce

Flight testing, flight operations and air transport

Captain W.D. Lowe, British Airways

Maintenance and airworthiness

G.B. Ratcliffe, RAeS Airworthiness and Maintenance Committee

Noise, aeroelasticity and fluid mechanics

Professor E.G. Broadbent, Department of Mathematics, Imperial College

Rotorcraft and structural dynamics

Professor D.E.H. Balmford, Westland Helicopters

\section{Space technology}

R. Gibson, Inmarsat

Structures and materials

T. Sharples, Military Aircraft Division, BAe Defence

Incorporating The Aeronautical Quarterly and the Journals

of The Institution of Aeronautical Engineers and

The Helicopter Association of Great Britain. 


\section{DIVISIONS AND BRANCHES OF}

\section{THE ROYAL AERONAUTICAL SOCIETY}

Australia Branches at: Adelaide, Brisbane, Canberra, Melbourne and Sydney

President: Air Cdre W.J. BELTON, CEng, FRAeS. Hon. Secretary: R.D. BARKLA, CEng, MRAeS. PO Box 573, Mascot, NSW 2020

Now Zealand Branches at: Auckland, Blenheim, Canterbury, Hamilton, Otago, Palmerston North and Weilington. President: Wg Cdr R.M. ALLAN, CEng, FRAeS, RNZAF Retd. Hon. Secretary; J.H. MOUNCE, CEng, MRAeS. Tech Service Manager - Int'l \& Dom, AKL35 Air New Zealand Limited, Private Bag,
Auckland 1.

Pakistan President: S. AJAZ ALI, CEng, FRAeS. Hon. Secretary: Dr S.A. HASAN, CEng, FRAeS, 4th Floor, IEP Building, 177/2 Liaquat Barracks, Karachi 75530. Tel: 92-7780233.

Southern Africa President: D.P. du PLOOY, CEng, MRAeS. Hon. Secretary. Ms LESLIE STOKOE, PO Box 9168,1625 Eldeen, Republic of South Africa.

Zlmbabwe President:Wg Cdr E. CHINGOSHO, MRAeS. Hon. Secretary: A.S. MATIKITI, IEng, AMRAeS. Engineering Dept., Air Zimbabwe, PO Box 1931, Harare, Zimbabwe.

\section{Bedford}

President: R.J. WHYSALL, CEng, MRAeS

Chairman: G.W. WOODS, CEng MRAeS.

Hon. Secretary: C.I.P. MARTIN, AMRAeS. Hunting Engineering, Reddings Wood, Ampthill, Bedford MK45 2HD. Tel: 0234841000

\section{Belfast}

President: T. STONE, FRAES.

Chairman: Professor D. NIXON, FRAeS.

Hon. Secretary: Dr S.T. MCILWAIN, Grad RAeS. Aerodynamics, Mechanical Engineering Group, Short Missile Systems, Montgomery Road, Belfast, Northern Ireland BT6 9HN. Tel: 0232465650

Birmingham, Wolverhampton and Cosford

President: S.L. HISCOX, MRAeS.

Chairman: R.J.P. HOWARD, AMRAES.

Hon. Secretary: A.R. BANBURY, CEng, Associate RAeS. 13 Grasmere Close, Tettenhall, Wolverhampton WV6 9DP. Tel: 0902745957.

\section{Boscombe Down}

President: Air Cdre C.J. CRUICKSHANKS, FRAeS.

Chairman: Dr D.W. WILLIAMS, CEng, FRAeS.

Hon. Secretary: Wg Cdr K. MACKENZIE, CEng, MRAES. ATG, Armament Division, A\&AEE Boscombe Down, Salisbury SP4 0JF. Tel: 0980662975.

\section{Bristol}

President: S.J. SWADLING, CEng, FRAES.

Chairman: D.C. GREENMAN, CEng, MRAeS

Hon. Secretary: J.G. MATTHEWS, Grad RAeS. Department B56, Technical Centre (C2) British Aerospace Airbus Ltd., Filton, Bristol BS99 7AR. Tel: 0272364006.

Brough

President: G. CHISNALL, CEng, MRAeS.

Chairman: S.J. BLEE, CEng, MRAeS

Hon. Secretary: G.M. CAMMISH. Manufacturing and Struc tural Development, BAe (Military Aircraft) Brough, North Humberside HU15 1EQ. Tel: 0482664143.

\section{Cambridge}

President:Professor M. GASTER, FRS, CEng, FRAeS.

Chairman: Wg Cdr P.N. GILBERT, CEng, MRAeS

Hon. Secretary: D.N. WHITEHEAD, CEng, MRAeS. c/o Aircraft Design Office, Marshall Aerospace, The Airport, Newrnarket Road, Cambridge CB5 8RX. Tel: 0223 $373232 / 373541$

\section{Cardiff}

President: Gp Capt I.F. EASTON, CEng, FRAeS.

Chairman: J A STEELE, IEng, AMRAeS.

Hon. Secretary: Wg Cdr A.G. WILLENBRUCH, CEng MRAeS. Engineering Wing, RAF St Athan, Barry CFE 9WA. Tel: 0446798798 Ex. 6053

\section{Chester}

President: S.A.E. DYKE

Chaiman: J.R.F. YOUNG, AMRAeS.

Hon. Secretary: Dr J.E. ROBINSON. Padeswood Lodge, Padeswood, Mold, Clwyd CH7 4JF. Tel: 0244546420

\section{Christchurch}

President: M.J. COBHAM, CBE, FRAeS.

Chairman: K.W. NORRIS, FRAeS.

Hon. Secretary: M.G. PORTER, CEng, MRAeS. Flight Refueling, Brook Road, Wimborne, Dorset BH21 2BJ. Tel: 0202882121 .

Coventry

President: J.B. ROBERTS CEng, FRAeS

Chairman: Dr B.D. HOBSON, CEng, MRAES.

Hon. Secretary: R.I. RATCLIFF, IEng, Technician RAeS. Erithway Rd, Green Lane, Coventry CV3 6JT. Tel: 0203 413779.

Cranwell

President: Air Vice Marshal D. COUSINS, RAF.

Chairman. Wg CdrM.F.J NETHAWAY.

Hon. Secretary: Sqn Ldr J.E. MORTON, CEng, MRAeS. RAF College Cranwell, Sleaford, Lincs NG34 8HB Tel: 0400261201 Ex. 6437 .

\section{Derby}

President: J.O. KEIR, FRAeS.

Chairman: D.C. COOK, CEng, MRAeS.

Hon. Secretary: K. COBLEY, CEng, MRAeS. c/o Compres sor Technology, Rolls-Royce plc, PO Box 31, Derby DE24 8BJ. Tel: 0332249017.

\section{Gatwick}

President: M.J. WILLETT, FRAeS.

Chairman: A.J. HEATH, OBE, CEng, FRAeS

Hon. Secretary: D.L. BATES, Associate RAeS. 52 Gran Road, Addiscombe, Croyden CR0 6PG. Tel: 081-654 1150 .
Glasgow

President: Dr A.W BABISTER, CEng, MRAeS.

Chairman: F.J. MULLEN.

on. Secretary: J. ALLAN, Grad RAeS. 47 Somerville Drive

Murray 7, East Kilbride G75 OLU. Tel: 0355263620.

Gloucester and Cheltenham

President: D.G.M. DAVIES, OBE, CEng, FRAeS.

Chairman: R.H. ASHFORTH, CEng, FRAeS.

Hon. Secretary: R.F.J. McCARTHY, CEng, FRAeS. Dowty

Aerospace, Anson Business Park, Cheltenham Road Gloucester GL2 9QH. Tel: 0452711428.

Hatfleld

President: F.W. VANN, CEng, FRAeS.

Chairman: F.B. OGILVIE, AMRAeS.

Hon. Secretary: D.H. TIPPER, CEng, MRAeS. 4 Bishops

Rise, Hatfield, Herts AL 10 9HB. Tel: 0707271025.

\section{Heathrow}

President: SIR COLIN MARSHALL, Hon Companion RAeS. Chairman: K.D. MITCHELL, Associate Member RAeS.

Hon. Secretary: R.A. KENNEDY, IEng, AMRAeS. British Airways (S.1) PO Box 10, Heathrow Airport, Hounslow, Middx TW6 2JA. Tel: 081-562 3366 .

\section{Isle of Wight}

President: R.L. WHEELER, CEng, FRAeS

Chairman: R.L. WHEELER, CEng, FRAeS.

Hon. Secretary: P.J. CHIVERS, CEng, MRAeS. Westland Aerospace, Castle Street, East Cowes, Isle of Wight PO32 6RH. Tel: 0983294101 Ex. 2016.

\section{Loughborough}

President: Professor G.L. WILDE, OBE, CEng, FRAeS.

Chairman: R.C.T. HARDING, Student RAES.

Hon. Secretary: Professor S.J. STEVENS, CEng, MRAeS. Transport Technology Dept, Loughborough University of Technology, Leics. LE11 3TU. Tel: 0509223403.

Luton

President: B. NEWTON

Chairman: P.J. SHARP, CEng, MRAeS

Hon. Secretary; N.G. TOOMEY, IEng, MRAeS. c/o Monarch Aircraft Engineering Ltd (Planning Dept), Luton International Airport, Luton, Beds LU2 YLX Tel: Work-0582 398542. Home-0234 54185.

\section{Manchester}

SCOTT-WILSON, OBE, FEng, FRAeS

Chairman: A.A. McDICKEN, MRAeS.

Hon. Secretary: I.R. FORD, Student RAeS. PS50, Avro International Aerospace, Woodford, Stockport, Cheshire SK7 IQR. Tel: 061-957 4586.

President: Gp Capt. G.E. STIRRUP, FRAeS.

Chairman: San Ldr R.B. ECKERSLEY, CEng, MRAeS.

Hon. Secretary: FIt Sgt N.P. KING, AMRAeS. 617 Squadron, RAF Marham, Kings Lynn, Nortolk PE33 9NP. Tel: 0760 337261 Ex. 6423 .

\section{Medway}

President: P.A. HEARNE, FEng, Hon. FRAeS.

Chairman: G.R. SLEIGHT, CEng, FRAeS.

Hon. Secretary: R.T. TWINE, MRAeS. c/o GEC-Marconi Avionics Ltd, Airport Works, Rochester, Kent ME1 $2 X X$ Tel: 0634844400 Ex. 3534

\section{Middle Wallop}

President: Col W.G. HARRISON.

Chairman: Lt Col T.W. PERKS, MRAeS.

Secretary: Major P. MILBORN, MBE, IEng, AMRAeS. OC Training Company, School of Aeronautical Engineering, Army Air Corps Centre, Middle Wallop, Stockbridge, Hampshire SO20 8DY. Tel: 0264384305.

Preston

President: Professor D. GARDNER, FEng, FRAeS.

Chairman: M. MANSELL, CEng, FRAeS

Hon. Secretary: M. GILCHRIST, BEng, Grad RAeS Weapons Management (W14), British Aerospace Defence, Warton Aerodrome, Preston, Lancs PR4 1AX. Tel: 0772855274.

Prestwick

President: P. BERRY, MRAeS.

Chairman: D.R. SHEPHERD, CEng, MRAeS

on. Secretary: A.R. FROST, AMRAeS. 21 Fullerton Road, Prestwick KA9 2BU. Tel: 029278204

\section{Sherborne}

President: Position vacant.

P. SAUNDERS, IEng AMRAeS. Hon. Secretary: J.D. SIMPSON, Student RAeS. 27 Mi
Close, East Coker, Yeovil BA22 9L.F. Tel: 0935702149.
Southampton

President: Professor G.M. LILLEY, CEng, FRAeS

Chairman: P.R. BOYLE, CEng, MRAeS.

Hon. Secretary: D.R.J. BAXTER, CEng, FRAeS. Department of Aeronautics and Astronautics, University of Southampton, Highfield, Southampton, Hants SO9 5NH. Tel: 0703 593882 .

Southend

President: BARONESS PLATT of WRITTLE, CBE, FEng, FRAeS.

Chairman: D. ROBERTS, Technician RAeS.

Hon. Secretary: EurIng M. NICOLINI, MRAeS. 29 Symons Avenue, Leigh-on-Sea, Essex SS9 5QD.Tel: 0702 521554 .

Stevenage

President:D A. LAYBOURN, FCA, FRAeS.

Chairman: Dr J.M. YOUNG, FRAES.

Hon. Secretary: D. MURPHY, CEng, MRAeS. British Aerospace Space Systems, Gunnels Wood Road, Stevenage, Herts SG1 2AS. Tel: 0438736737

\section{Swindon}

President: Professor M.G. FARLEY, CEng, FRAeS.

Chairman: Wg Cdr T. KIRBY

Hon. Secretary: V.A. SIMON, 20 Hawkswood, Covingham

Park, Swindon, Wilts. Tel: 0793525350.

\section{Weybridge}

President: Professor I.R. YATES, CBE, FEng, FRAeS.

Chairman: Miss J.M. GULLAND CEng, MRAeS.

Hon. Secretary: K.J. DAVIES, CEng, MRAeS, 65 Stringers Avenue, Jacobs Well, Guildford, Surrey GU4 7NN. Tel: 048331529.

Yeovil

President: V.A.B. ROGERS, CBE, FEng, FRAeS.

Chairman: R.I. CASE, FEng, FRAeS.

Hon. Secretary: F.N.R. BALLAM, CEng, MRAeS. Box 285 , Westland Helicopters, Yeovil, Somerset BA20 2YB. Tel: 0935703779

\section{OVERSEAS BRANCHES}

Cyprus

President: D.M. PANTAZIS, FRAeS.

Chairman: D.M. PANTAZIS, FRAES

Hon. Secretary: C. PERICLEOUS, MRAeS. 8 Georgiou Christodoulou, Acropolis, Nicosia, Cyprus. Tel: $357-2$ 310430 .

Hong Kong

Chairman: R. FAIRFIELD, FRAeS

Hon. Secretary: M.J. POMFRET, MRAeS. MME Dept HK Polytechnic, Kowloon, Hong Kong. Tel: Office 37666647. Home: 3-7213732.

Malaysia

Chairman: Dato R. SINGH, IEng, FRAeS.

Hon. Secretary: Lt Col A. LATIF B. TAHA, AMRAeS, MRAF, Dept of the Air Force, Ministry of Defence, Jalan Padang Tembak, 50634 Kuala Lumpur, Malaysia. Tel: 03-2354358.

Munich

President: Professor Dipling G. MADELUNG, FEng, HonFRAes.

Chairman: J. VINCENT, FRAeS.

Hon. Secretary: G. PARKER, Student RAeS. NAMMA, Postfach 1303, B2003 Unterhaching, Germany. Tel: 89-666 80140 .

Northern Germany

Chairman: Sqn Ldr A.C. MAJOR, CEng, MRAeS, RAF.

Hon Secretary: Sqn Ldr A.C. MAJOR, CEng, MRAeS, RAF. Puma Maintenance FIt, RAF Laarburch, BFPO 43, Germany. Tel: 02837895367

\section{Republic of Ireland}

President: R.W. O'SULLIVAN, CEng, FRAeS.

Chairman: H. REILLY, MRAES.

Hon. Secretary: J.J. CONERNEY, Technician RAeS. Eng. Wing, Air Corps, Baldonnel, Co. Dublin. Tel: 592493. Ex. 6633 .

Toulouse

Presidemt: J. PIERSON, FRAeS.

Chairman: A.S. McCLYMONT, FRAeS.

Hon. Secretary: W.A.N. FORD, CEng, FRAeS. Chrestias, Avenue du Bouconne, 31530 Lévignac, France. Tel: (33) 61854258 . 